\title{
Hubble Space Telescope Imaging of Antlia B: Star Formation History and a New Tip of the Red Giant Branch Distance
}

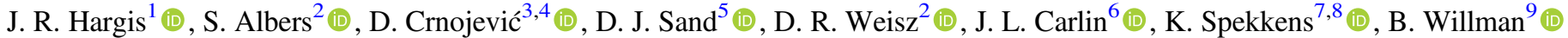 \\ A. H. G. Peter $^{10}$ (D) C. J. Grillmair ${ }^{11}$, and A. E. Dolphin ${ }^{12}$ (D) \\ ${ }^{1}$ Space Telescope Science Institute, 3800 San Martin Drive, Baltimore, MD 21208, USA; jhargis@ stsci.edu \\ ${ }^{2}$ University of California, Berkeley, Department of Astronomy, 501 Campbell Hall \#3411, Berkeley, CA 94720-3411, USA \\ ${ }^{3}$ University of Tampa, 401 West Kennedy Boulevard, Tampa, FL 33606, USA \\ ${ }^{4}$ Department of Physics \& Astronomy, Texas Tech University, Box 41051, Lubbock, TX 79409-1051, USA \\ ${ }^{5}$ Steward Observatory, University of Arizona, 933 North Cherry Avenue, Rm. N204, Tucson, AZ 85721-0065, USA \\ ${ }^{6}$ LSST, 950 North Cherry Avenue, Tucson, AZ 85719, USA \\ ${ }^{7}$ Department of Physics and Space Science, Royal Military College of Canada P.O. Box 17000, Station Forces Kingston, ON K7K 7B4, Canada \\ ${ }_{9}^{8}$ Department of Physics, Engineering Physics and Astronomy, Queen's University, Kingston, ON K7L 3N6, Canada \\ ${ }^{9}$ NSF's National Optical-Infrared Astronomy Research Laboratory, 950 N Cherry Avenue, Tucson, AZ 85721, USA \\ ${ }^{10}$ CCAPP, Department of Physics, and Department of Astronomy, The Ohio State University, Columbus, OH 43210, USA \\ ${ }^{11}$ IPAC, California Institute of Technology, Pasadena, CA 91125, USA \\ ${ }^{12}$ Raytheon Company, Tucson, AZ 85734, USA \\ Received 2019 August 2; revised 2019 October 25; accepted 2019 November 5; published 2020 January 3
}

\begin{abstract}
A census of the satellite population around dwarf galaxy primary hosts in environments outside the Local Group is essential to understanding $\Lambda$ cold dark matter galaxy formation and evolution on the smallest scales. We present deep optical Hubble Space Telescope imaging of the gas-rich, faint dwarf galaxy Antlia B $\left(M_{V}=-9.4\right)$-a likely satellite of NGC $3109(D=1.3 \mathrm{Mpc}$ )-discovered as part of our ongoing survey of primary host galaxies similar to the Magellanic Clouds. We derive a new tip of the red giant branch distance of $D=1.35 \pm 0.06 \mathrm{Mpc}$ ( $m-M=25.65 \pm 0.10$ ), consistent with membership in the nearby NGC 3109 dwarf association. The colormagnitude diagram (CMD) shows both a prominent old, metal-poor stellar component and confirms a small population of young, blue stars with ages $\lesssim 1$ Gyr. We use the CMD fitting algorithm MATCH to derive the star formation history (SFH) and find that it is consistent with the typical dwarf irregular or transitional dwarf galaxy (dTrans) in the Local Group. Antlia B shows relatively constant stellar mass growth for the first $~ 10-11 \mathrm{Gyr}$ and almost no growth in the last 2-3 Gyr. Despite being gas-rich, Antlia B shows no evidence of active star formation (i.e., no $\mathrm{H} \alpha$ emission) and should therefore be classified as a dTrans dwarf. Both Antlia B and the Antlia dwarf (dTrans) are likely satellites of NGC 3109, suggesting that the cessation of ongoing star formation in these galaxies may be environmentally driven. Future work studying the gas kinematics and distribution in Antlia B will explore this scenario in greater detail. Our work highlights the fact that detailed studies of nearby dwarf galaxies in a variety of environments may continue to shed light on the processes that drive the SFH and evolution of dwarf galaxies more generally.
\end{abstract}

Unified Astronomy Thesaurus concepts: Dwarf galaxies (416); Dwarf irregular galaxies (417); Star formation (1569); Hubble Space Telescope (761)

Supporting material: machine-readable tables

\section{Introduction}

The $\Lambda$ cold dark matter $(\Lambda \mathrm{CDM})$ model for structure formation is very successful at describing the universe on large scales ( $\gtrsim 10 \mathrm{Mpc}$ ), but continues to face challenges on smaller, subgalactic scales (see Bullock \& Boylan-Kolchin 2017, for a recent review), where several "problems" with the faint end of the galaxy luminosity function (LF) manifest themselves. Work on both theoretical (e.g., Brooks et al. 2013; Sawala et al. 2016; Wetzel et al. 2016; Kim et al. 2018) and observational (e.g., Koposov et al. 2018; Torrealba et al. 2018) fronts has primarily focused on reconciling $\Lambda \mathrm{CDM}$ issues in the context of the Milky Way and its satellite system. However, to truly test the $\Lambda \mathrm{CDM}$ model for structure formation on the smallest scales, observational studies of satellite populations beyond the Local Group are necessary and must sample primary halos with a range of masses, morphologies, and environments. This work is advancing with a primary focus on Milky Way-like galaxies in the Local Volume (e.g., Chiboucas et al. 2009; Crnojević et al. 2014, 2016, 2019; Sand et al. 2014, 2015; Carlin et al.
2016, 2019; Toloba et al. 2016; Bennet et al. 2017, 2019; Carrillo et al. 2017; Danieli et al. 2017; Geha et al. 2017; Smercina et al. 2017, 2018).

One opportunity to explore $\Lambda \mathrm{CDM}$ on smaller scales is to survey the satellite population around low-mass host galaxies, similar to the Magellanic Clouds, which recent work has suggested has its own satellite system (e.g., Sales et al. 2017; Kallivayalil et al. 2018). Systematic searches of this kind would not only shed light on the local LMC/SMC system, but may also help tease out the roles that environment (e.g., rampressure or tidal stripping) and primary host galaxy mass play in shaping a satellite system (e.g., Gatto et al. 2013; Dooley et al. 2017). Our survey program has published initial results for two systems: NGC $3109(D=1.3 \mathrm{Mpc}$; $\left.M_{*} \approx 7 \times 10^{8} M_{\odot}\right)$ and NGC $2403\left(D=3.2 \mathrm{Mpc} ; M_{*} \approx 7 \times\right.$ $\left.10^{9} M_{\odot}\right)$. Each search turned up new, faint dwarf galaxiesAntlia B around NGC 3109 (Sand et al. 2015) and NGC 2403Dw1 around NGC 2403 (Carlin et al. 2016).

Utilizing galaxy satellite populations as probes of smallscale cosmological structure requires not only discovering new 
Table 1

Properties of Antlia B

\begin{tabular}{|c|c|c|}
\hline Parameter & Value & Source \\
\hline R.A.o (h:m:s) & $09: 48: 56$ ". $^{\prime \prime} \pm 2$ ". 1 & Sand et al. (2015) \\
\hline $\operatorname{Decl}_{.}(\mathrm{d}: \mathrm{m}: \mathrm{s})$ & $-25: 59: 24 ! \prime 0 \pm 3 " .8$ & Sand et al. (2015) \\
\hline$(m-M)_{0}(\mathrm{mag})$ & $25.65 \pm 0.10$ & This work \\
\hline$D(\mathrm{Mpc})$ & $1.35 \pm 0.06$ & This work \\
\hline$D_{\text {proj }}(\mathrm{kpc})$ & 73 & $\ldots$ \\
\hline$M_{V}(\mathrm{mag})$ & $-9.7 \pm 0.6$ & Sand et al. (2015) \\
\hline$r_{\text {half }}(\operatorname{arcsec})$ & $43.2 \pm 4.2$ & Sand et al. (2015) \\
\hline$r_{\text {half }}(\mathrm{pc})$ & $273 \pm 29$ & Sand et al. (2015) \\
\hline$\epsilon$ & $0.30 \pm 0.05$ & Sand et al. (2015) \\
\hline$\theta(\operatorname{deg})$ & $4.0 \pm 12.0$ & Sand et al. (2015) \\
\hline$S_{21}\left(\mathrm{Jy} \mathrm{km} \mathrm{s}^{-1}\right)$ & $0.72 \pm 0.05$ & Sand et al. (2015) \\
\hline$W 50_{\mathrm{H} \mathrm{I}}\left(\mathrm{km} \mathrm{s}^{-1}\right)$ & $17 \pm 4$ & Sand et al. (2015) \\
\hline$M_{\mathrm{H} \mathrm{I}}\left(10^{5} M_{\odot}\right)$ & $2.8 \pm 0.2$ & Sand et al. (2015) \\
\hline$v_{\text {helio, } \mathrm{H} \mathrm{I}}\left(\mathrm{km} \mathrm{s}^{-1}\right)$ & $376 \pm 2$ & Sand et al. (2015) \\
\hline
\end{tabular}

satellites but also understanding galaxy formation and evolution in the dwarf galaxy regime; that is, how baryons populate dark matter halos at small scales and how physical processes shape the present-day, observed properties of dwarf galaxies. Studies of the resolved stellar populations of dwarf galaxiesvia color-magnitude diagrams (CMDs) and star formation histories (SFHs) - have been essential observational tools for understanding dwarf galaxy evolution (e.g., Mateo 1998; Tolstoy et al. 2009; Weisz et al. 2011, and references therein). In the last decade, a combination of deep optical CMDs from the Hubble Space Telescope (HST) and increasingly sophisticated stellar evolution models (e.g., Dotter et al. 2008; Girardi et al. 2010; VandenBerg et al. 2014; Choi et al. 2016; Marigo et al. 2017) have provided a systematic census of the SFHs of dwarf galaxies within $\sim 3 \mathrm{Mpc}$ (e.g., McQuinn et al. 2010, 2015a; Weisz et al. 2011, 2014; Skillman et al. 2017).

In this paper, we focus on deep optical HST observations of Antlia B to follow-up on the ground-based discovery of this gas-rich, faint dwarf galaxy at $D=1.3 \mathrm{Mpc}$. For reference, we list many of the properties of Antlia B in Table 1, including position, half-light radius ( $\left.r_{\text {half }}\right)$, absolute magnitude $\left(M_{V}\right)$ and single-dish H I gas properties. Most of these properties were derived in Sand et al. (2015), and are used in the current work because the incomplete spatial coverage of HST does not allow us to significantly update these parameters. In Section 2 we discuss the observations and data reduction, and in Section 3 we present the CMD. We present an updated tip of the red giant branch (TRGB) distance to Antlia B in Section 4, as well as a quantitative $\mathrm{SFH}$ in Section 5 . We conclude the paper by putting Antlia B in context with the other dwarf galaxies in the NGC 3109 association-NGC 3109 itself, Antlia, Sextans A, Sextans B, and Leo P-as well as that of the Local Group (Section 6).

\section{HST Observations}

Observations of Antlia B were taken under HST program HST-GO-14078 (PI: J. Hargis) on 2017 January 4 with the Advanced Camera for Surveys (ACS, Ford et al. 1998), using the Wide Field Channel. One HST orbit of observations was taken in the F606W and F814W filters, with total exposure times (from two images) of 934 and $1142 \mathrm{~s}$, respectively. A two-point dither was taken for each filter to help with cosmic ray and hot pixel removal. A color composite of one of the
ACS chips is shown in Figure 1 overlaid on a cutout of the discovery DECam image (Sand et al. 2015). The HST panel clearly shows the overdensity of stars associated with Antlia B. The ACS field of view was oriented so that the bright star to the North of Antlia B was off the chip; inevitably, this means that some Antlia B stars were not on the ACS chip, and bleed trails from the bright star affect some stellar photometry at the edge of the field of view. This does not affect our main science goals to measure the distance and SFH of the dwarf.

The imaging data were reduced using the CALACS pipeline (Version 8.3.5) and retrieved from the Mikulski Archive for Space Telescopes (MAST). All the HST data used in this paper can be found in MAST here: doi: 10.17909/t9-ata8-2294. Point-spread function photometry was performed on the CTEcorrected .flc images with the DOLPHOT v2.0 photometry package, a version of HSTPHOT (Dolphin 2000) that has been modified for use with ACS. We reduced the data using the DOLPHOT parameters and pre- and post-processing steps prescribed in Williams et al. (2014). To construct our final list of good stars, we culled the raw photometric catalogs, keeping only sources that passed the following measurement criteria: $\operatorname{crowd}_{\mathrm{F} 606 \mathrm{~W}}+\operatorname{crowd}_{\mathrm{F} 814 \mathrm{~W}}<1, \quad \operatorname{sharp}_{\mathrm{F} 606 \mathrm{~W}}^{2}+\operatorname{sharp}_{\mathrm{F} 814 \mathrm{~W}}^{2}<$ $0.1, \mathrm{~S} / \mathrm{N}_{\mathrm{F} 606 \mathrm{~W}}>5$, and $\mathrm{S} / \mathrm{N}_{\mathrm{F} 814 \mathrm{~W}}>5$. A small number of spurious source detections around bright or saturated objects were removed manually. There is no evidence for crowding, even at the center of Antlia B.

We performed artificial star tests (ASTs) in order to quantify the photometric errors and incompleteness in our observations. A total of 500,000 ASTS, implanted one star at a time, were distributed uniformly both in color-magnitude space (i.e., across the relevant region of the CMD) and spatially across the field of view to avoid crowding. ASTs were injected up to 2 mag fainter than the faintest detected stars in order to adequately sample regions of low completeness. Photometry and quality cuts were performed in an identical manner to those performed on the original photometry. Photometric errors are shown as a function of F814W magnitude at the approximate color of the Antlia B ridge line in Figure 2. We are 50\% (90\%) complete in F814W at 26.71 (26.31) and in F606W at $\sim 27.40$ (27.00) mag.

We present our final photometric catalog of resolved stars in Table 2. The calibrated F814W and F606W magnitudes listed in the table are uncorrected for Galactic extinction. In addition to the full DOLPHOT output, we also include F606W and F814W Milky Way extinction values on a star by star basis, using the dust maps of Schlegel et al. (1998) and coefficients of Schlafly \& Finkbeiner (2011). All CMDs presented in this work have been extinction-corrected with these values. For reference, Antlia B has a typical color excess of $E$ $(B-V) \approx 0.080 \mathrm{mag}$.

\section{Color-Magnitude Diagram and Stellar Population Spatial Variations}

The CMD within the half-light radius $\left(r_{\text {half }}=273\right.$ pc or 43". 2) of Antlia B is shown in Figure 2(a). Antlia B has both a significant old, metal-poor red giant branch (RGB) population and a prominent intermediate age, more metal-rich red clump (RC) population. For comparison, in Figure 2(b) we show theoretical isochrones for old, metal-poor populations (age $=13.5 \mathrm{Gyr} ;[\mathrm{Fe} / \mathrm{H}]=-1.5,-2)$ from Dotter et al. (2008) and young, more metal-rich populations (ages 250, 400, $700 \mathrm{Myr}$; $[\mathrm{Fe} / \mathrm{H}]=-1$ ) from Marigo et al. (2017). 


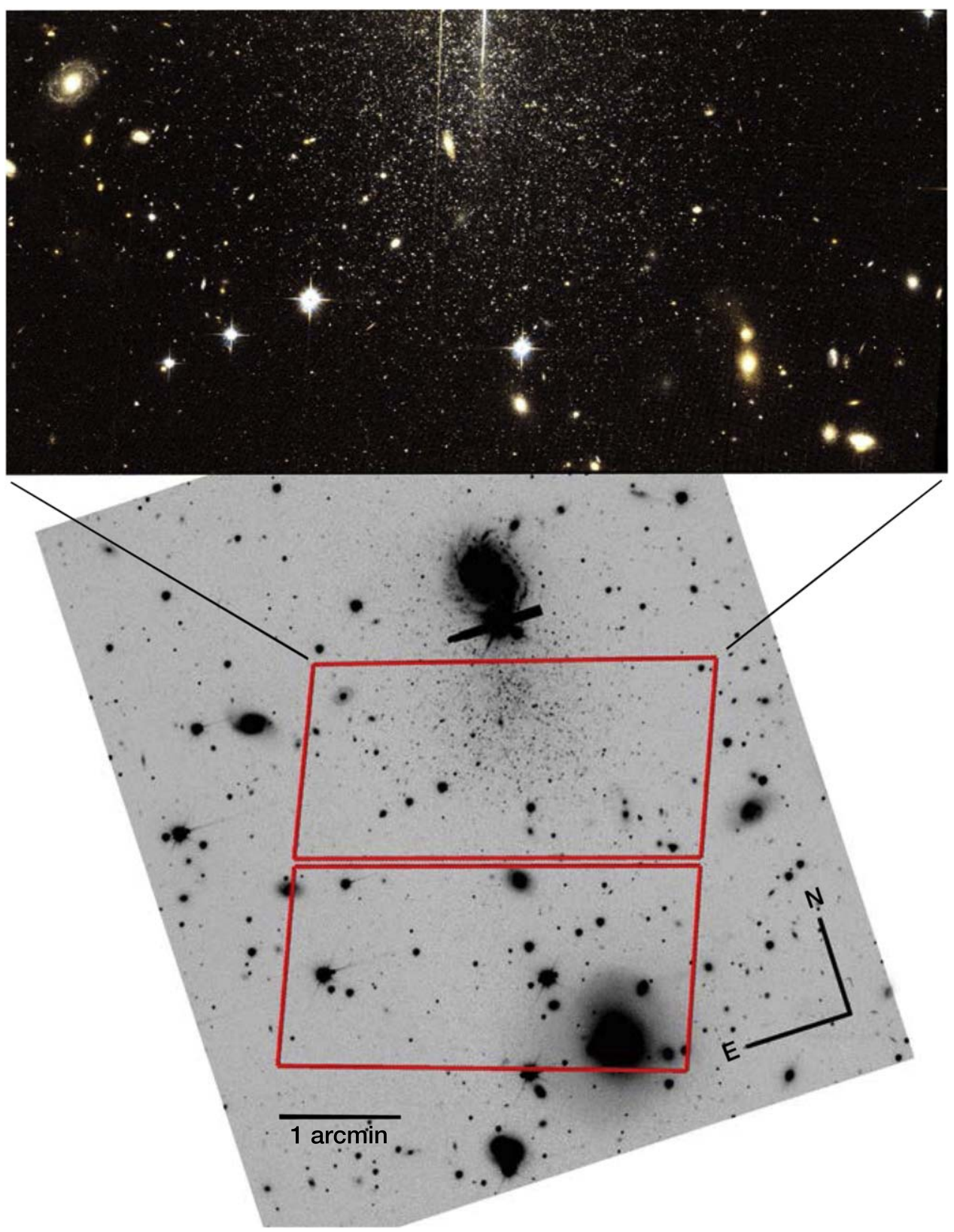

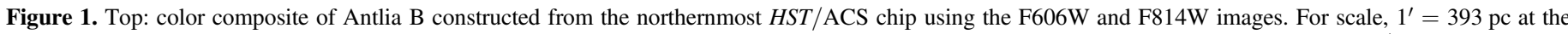

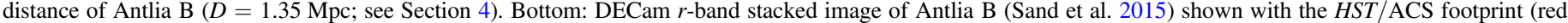

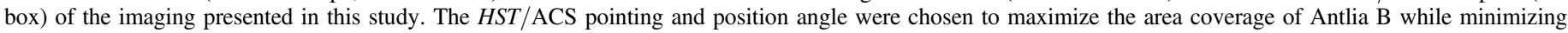
contamination from the bright foreground star and the background galaxy north of Antlia B. The HST data clearly show the stellar overdensity that is Antlia B.

Antlia B shows evidence of a small population of relatively bright, blue stars at $\mathrm{F} 606 \mathrm{~W}-\mathrm{F} 814 \mathrm{~W} \approx 0$, consistent with a small population of young main-sequence stars with ages of $<1$ Gyr (see blue selection box in Figures 2(b)-(d)). We find no evidence for very recent star formation (ages $\lesssim 10 \mathrm{Myr}$ ), consistent with the ground-based $\mathrm{H} \alpha$ imaging from Sand et al. (2015).

To explore the spatial variations in the stellar populations of Antlia B, we define four spatial regions of equivalent area: one elliptical region enclosing $r_{\text {half }}$, one ellipse just outside of the half-light radius enclosing $r_{\text {half }}<r<1.5 r_{\text {half }}$, and two boxes in representative "background" regions at projected radii between $r \sim 2.5-3 r_{\text {half }}$ from the galaxy center. The ellipse shapes are based on the structural analysis of Antlia B from the ground-based imaging by Sand et al. (2015; see Table 1). A first background region was chosen along the major axis of Antlia B at a radial distance $r \sim 3 r_{\text {half. }}$ A second background region was chosen away from Antlia B to avoid possible contamination from the overdensity of sources around the background galaxy cluster (AS0620A) to the southwest of Antlia B.

Figure 3 shows the regions overlaid on the HST field of view and the corresponding CMDs are shown in Figure 2. The blue stellar component is clearly spatially concentrated within the inner $r_{\text {half }}$. Outside of $r_{\text {half }}$ there are significantly fewer blue sources; the majority of these are faint $(\mathrm{F} 814 \mathrm{~W}>25.5)$, have correspondingly larger photometric color uncertainties, and therefore cannot be confidently associated with Antlia B. Regardless, the strong central concentration of sources suggests that these objects are indeed a younger, more metal-rich 

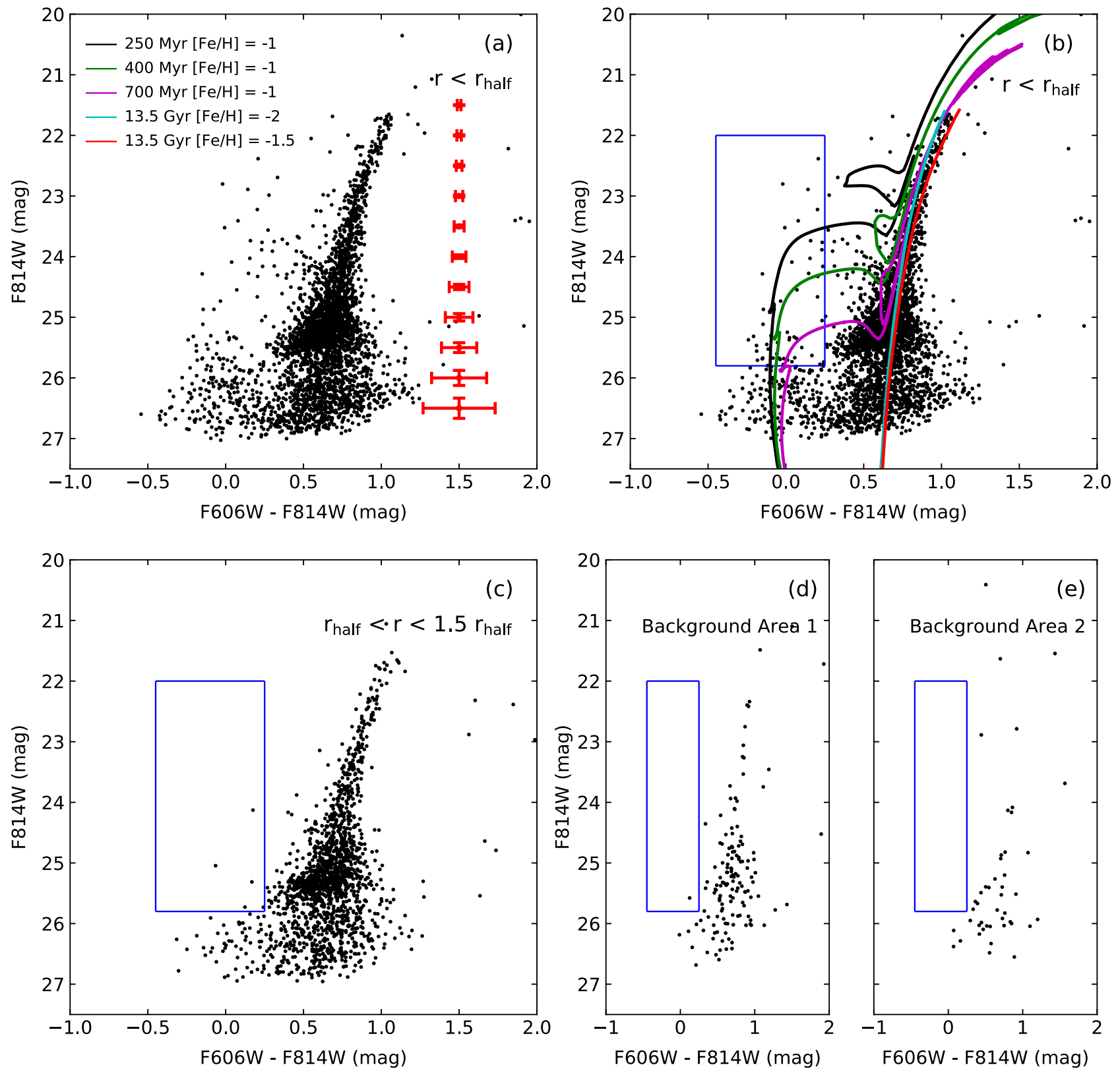

Figure 2. Optical CMDs of Antlia B. (a) Point sources within the half-light radius of Antlia B are shown in black. The median color (F606W - F814W) and F814W magnitude errors are shown as a function of magnitude as red points. (b) Same as panel (a) but showing theoretical isochrones for a range of stellar metallicities and ages (see Section 3). The blue box highlights the relatively bright, blue sources at F606W - F814W $\simeq 0$. These are consistent with a younger, more metal-rich mainsequence stellar population. Panel (c) show the CMD of the the outer regions Antlia B ( $r_{\text {half }}<r<1.5 r_{\text {half }}$; see Figure 3 ). As indicated by the blue selection box, the young, blue stellar populations show a centrally concentrated spatial distribution. For comparison, panels (d) and (e) show the CMDs derived from two equal-area background regions marked in Figure 3.

population in Antlia B and not the result of contamination from unresolved background galaxies or old blue horizontal branch stars. Furthermore, we note that the majority of the centrally concentrated blue sources are clustered with an arc-shaped distribution slightly off-center.

Sampling regions at larger galactocentric radii $\left(r \gtrsim 1.5 r_{\text {half }}\right)$ shows that while the surface density of stars is lower, there is clear evidence for Antlia B stars out to $\sim 3 r_{\text {half }}$. In Figure 2(d), the CMD of background area 1 (see Figure 3) shows a clear $\mathrm{RGB}$, consistent with the CMD of the central region of Antlia B. In contrast, the region offset from Antlia B (background area 2; see Figure 3) shows no significant structure in the CMD (Figure 2(e)), although we note that the surface density of sources is higher in background area 1.

A concentrated spatial distribution of younger stars or starforming regions relative to an extended distribution of older stars is typical for dwarf galaxies in the Local Volume (see Stinson et al. 2009, and references therein). For galaxies in the NGC 3109 dwarf association in particular the oldest stellar components (age $\gtrsim 5 \mathrm{Gyr}$ ) show smooth, extended spatial distributions in contrast to more recent star formation (age $\lesssim 1 \mathrm{Gyr}$ ). Sextans A has a patchy distribution of young/ intermediate age stellar populations $(\sim 50-700 \mathrm{Myr})$, but has a smooth extended spatial component as traced by the old RGB 
Table 2

Photometry of Resolved Stars in the HST/ACS Imaging of Antlia B

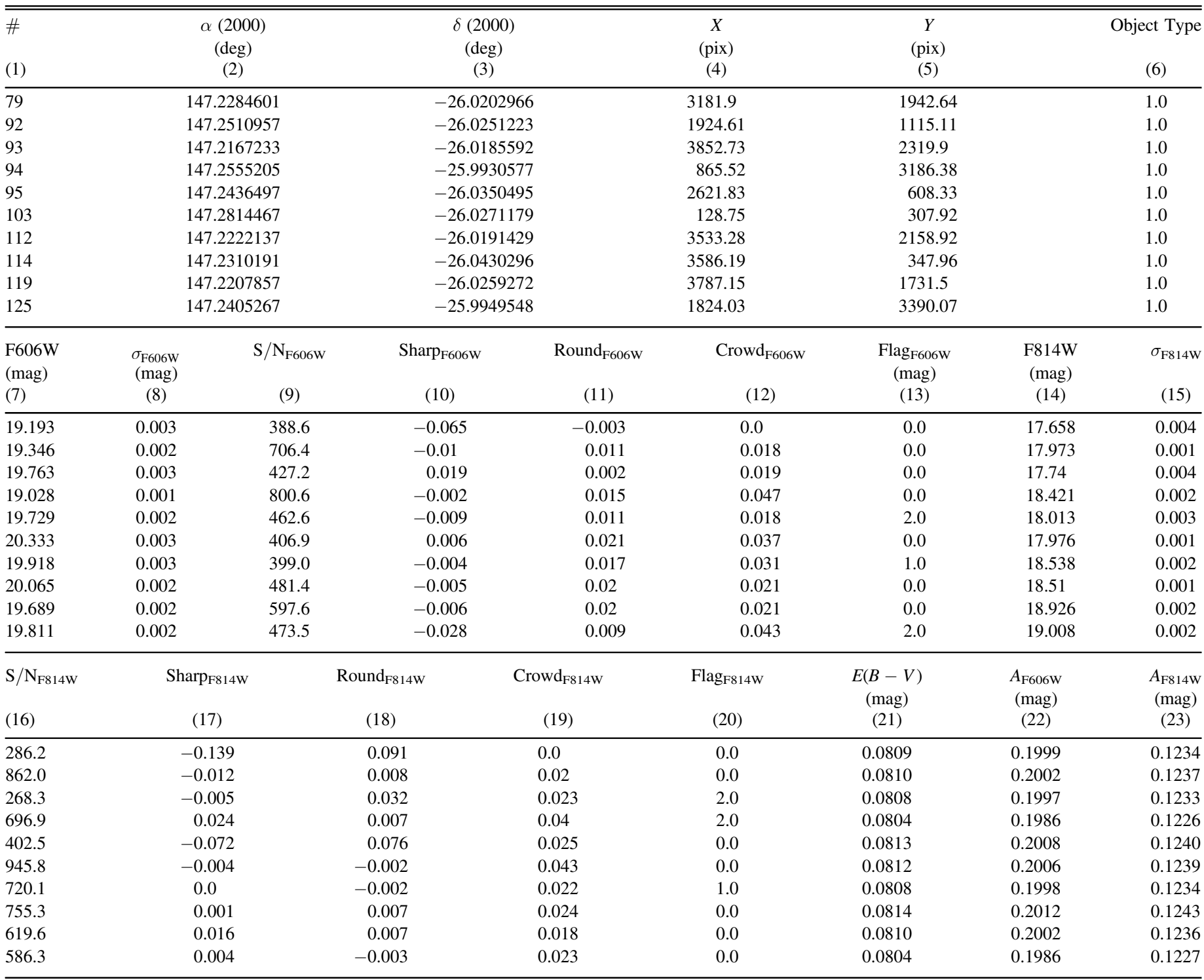

Note. Photometric catalog of resolved stars in the HST/ACS data set used in this study. Sources that did not pass the point-source selection criteria described in Section 2 were not included in this catalog. For completeness, we provide the full output from our DOLPHOT photometry and refer the reader to the DOLPHOT documentation for specific details on the column descriptions. Note that the photometry in this table is not corrected for Milky Way extinction, but extinction values are provided for convenience. All figures in this paper have been correct for Galactic extinction using Columns (21)-(23) described below. (1) Object identification number. (2)-(5) Position of sources in the celestial equatorial and image frames of reference. (6) Source object type as described by DOLPHOT. (7)-(13) Calibrated magnitudes, errors, signal-to-noise (S/N), shape parameters (sharp, round), crowding parameter, and quality flag for the F606W photometry. (14)-(20) Same as for columns (7)-(13) but for the F814W photometry. (21) Color excess for each source from Schlegel et al. (1998) dust maps. (22)-(23) Milky Way extinction values in F606W and F814W filters derived using the coefficients from Schlafly \& Finkbeiner (2011).

(This table is available in its entirety in machine-readable form.)

population (van Dyk et al. 1998; Dohm-Palmer et al. 2002; Dolphin et al. 2003; Bellazzini et al. 2014). Sextans B shows a similar spatial structure between young and old populations, albeit with a smaller rate of current star formation (Weisz et al. 2011; Bellazzini et al. 2014). NGC 3109 also has centrally concentrated regions of young blue stars (ages $\lesssim 1 \mathrm{Gyr}$ ) and a spatially extended old RGB population (Minniti et al. 1999; Hidalgo et al. 2008; Weisz et al. 2011). The younger population of stars in the Antlia dwarf are also centrally concentrated relative to the extended population of older, metal-poor stars (Penny et al. 2012). Lastly, Leo P has at least one active region of star formation (e.g., single O-star and $\mathrm{H}$ II region; Skillman et al. 2013; Evans et al. 2019) in the central region of the galaxy, while the older RGB population shows a larger spatial extent (McQuinn et al. 2015b).

\section{TRGB Distance}

The TRGB magnitude value is an excellent distance indicator for nearby galaxies resolved into stars (e.g., Lee et al. 1993; Sakai et al. 1997; Makarov et al. 2006; Rizzi et al. 2007). Sand et al. (2015) used the $r$-band DECam imaging of 


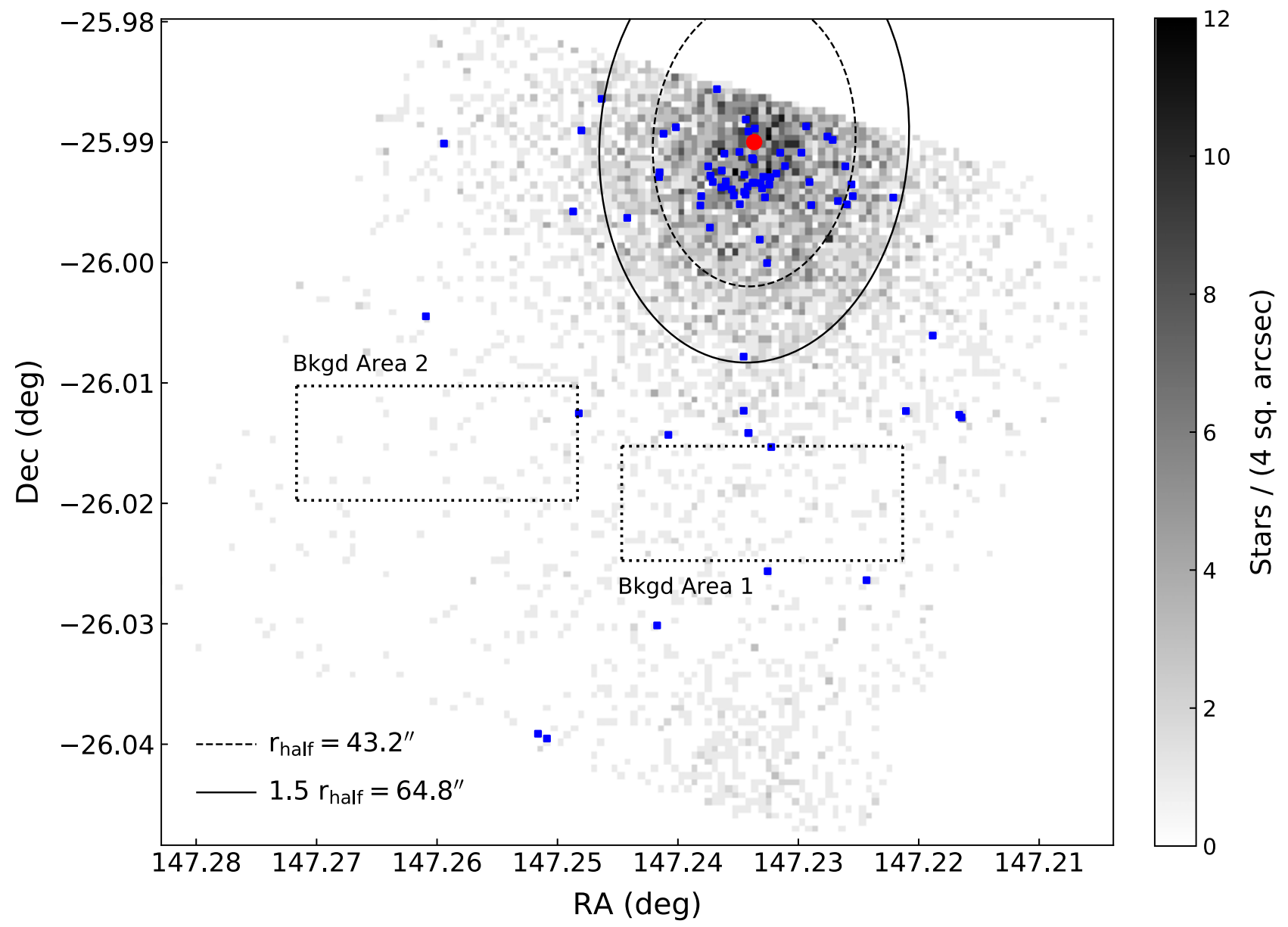

Figure 3. Spatial distribution of the Antlia B stellar populations. The grayscale bins show the surface density of all stars brighter than the $90 \%$ completeness limit (F814W < 26.3; see Figure 2). The blue points denote the individual relatively bright, blue stars in the selection box shown in Figure 2. The bluer stellar populations show a strong central concentration relative to the more extended old, metal-poor RGB and RC populations. The red dot denotes the galaxy center as measured in the ground-based imaging (Sand et al. 2015). The dashed ellipse is drawn with the semimajor axis equal to the half-light radius as measured by Sand et al. (2015, $r_{\text {half }}=43^{\prime \prime} .2=273 \mathrm{pc}, \epsilon=0.3, \mathrm{PA}=4^{\circ}$ ). An annular region between 1 and $1.5 r_{\text {half }}$ (solid line) encloses an area identical to that within $r_{\text {half }}$, taking into account the missing area of the HST pointing. The two dotted squares denote background areas of identical area for comparison. The CMDs of stars within these regions are shown in Figure 2.

Antlia B to obtain a TRGB distance of $D=1.29 \pm 0.10 \mathrm{Mpc}$ $\left(m-M_{0}=25.56 \pm 0.16 \mathrm{mag}\right)$. We redetermine the TRGB distance here with the HST/ACS data set, which has both higher signal-to-noise ratio $(\mathrm{S} / \mathrm{N})$ and superior star-galaxy separation than the ground-based data.

We adopt the TRGB absolute magnitude calibration in the F814W filter from Jang \& Lee (2017):

$$
\begin{aligned}
& M_{\mathrm{F} 814 \mathrm{~W}}^{\mathrm{TRGB}}=-4.015( \pm 0.056)-0.159( \pm 0.01) \\
& \quad \times\left[(\mathrm{F} 606 \mathrm{~W}-\mathrm{F} 814 \mathrm{~W})_{0}-1.1\right]^{2}+0.047( \pm 0.02) \\
& \quad \times\left[(\mathrm{F} 606 \mathrm{~W}-\mathrm{F} 814 \mathrm{~W})_{0}-1.1\right] .
\end{aligned}
$$

We apply the metallicity-dependent color correction term from the absolute magnitude formula (1) to our extinctioncorrected photometry to obtain a more well defined measure of the TRGB (see, e.g., Madore et al. 2009; McQuinn et al. 2016). We adopt the approach of Makarov et al. (2006) to find the TRGB, where a pre-defined LF is compared to the observed RGB LF. The model LF has the form

$$
\psi= \begin{cases}10^{a\left(m-m_{\mathrm{TRGB}}\right)+b}, & m-m_{\mathrm{TRGB}} \geqslant 0 \\ 10^{c\left(m-m_{\mathrm{TRGB}}\right)}, & m-m_{\mathrm{TRGB}}<0\end{cases}
$$

where $a$ and $c$ are the slopes of the RGB and AGB, respectively, and $b$ represents the discontinuity at the TRGB magnitude. The model LF is convolved with the photometric uncertainty, bias, and completeness function derived from the ASTs, and subsequently fit with a nonlinear least-squares (Levenberg-Marquardt) method for increased computational speed. As an initial guess for the algorithm, we estimated $m_{\text {TRGB }}$ using the results of a Sobel edge-detection filter (see Sakai et al. 1997; Crnojević et al. 2019 for details). In general we find that the model-fitting TRGB method provides a more robust distance estimate and smaller uncertainties than the Sobel filter, primarily because the latter method is sensitive to the choice of the LF bin size.

We derive a value of $m_{\mathrm{TRGB}}=21.63 \pm 0.08$, corresponding to a TRGB distance of $D=1.35 \pm 0.06 \mathrm{Mpc}\left((m-M)_{0}=\right.$ $25.65 \pm 0.10 \mathrm{mag}$ ). This is $\sim 0.05 \mathrm{Mpc}$ more distant than the ground-based TRGB distance from Sand et al. (2015), consistent with their result within the uncertainties. Considering the distance and projected separation $\left(D_{\text {proj }}=73 \mathrm{kpc}\right)$ of Antlia B from NGC $3109\left(D_{\mathrm{TRGB}}=1.28 \pm 0.03 \mathrm{Mpc}\right.$; Dalcanton et al. 2009), it is clear that Antlia B is associated with 
NGC 3109-either as a bound satellite or member of the broader NGC 3109 dwarf association. Given the distance uncertainties, whether or not Antlia B lies within the virial radius of NGC 3109 remains an open question. As discussed by Sand et al. (2015), if Antlia B lies within the virial volume of NGC $3109(\sim 100 \mathrm{kpc})$ one might expect that ram-pressure stripping (or other physical mechanisms) may have removed the gas from Antlia B (see additional discussion in Section 6).

\section{Star Formation History}

We measure the quantitative SFH of Antlia B using MATCH (Dolphin 2002) following implementations detailed in the literature (e.g., Weisz et al. 2011, 2014). Here, we briefly summarize.

MATCH generates a model CMD given specified parameters including IMF slope, binary fraction, distance, extinction, age and metallicity bin widths, and a given set of stellar models. It constructs a composite model CMD, which is then convolved with the error distribution and completeness function measured from ASTs. A foreground component is added to create a final model CMD. This model CMD is compared to the observed CMD using a Poisson likelihood function. The code computes multiple realizations of the SFH (i.e., by varying weights on each age and metallicity bin) and reevaluates the likelihood function until a maximum likelihood solution is found.

In the case of Antlia B, we adopted parameters identical to those used in Weisz et al. (2014): a Kroupa IMF (Kroupa 2001), a binary fraction of 0.35 with a uniform mass ratio, the Padova stellar evolution models (Girardi et al. 2010), a metallicity grid ranging from $-2.3 \leqslant[\mathrm{M} / \mathrm{H}] \leqslant-0.1$ with a resolution of 0.1 and an age grid of $\log (t)=10.15-9.00$ in steps of $\Delta \log$ $(t)=0.05$ and $\log (t)=9.00-6.60$ in steps of $\Delta \log (t)=0.05$. We adopt the TRGB distance as measured in Section 4 and the Milky Way foreground extinction values from Schlafly \& Finkbeiner (2011) at the position of Antlia B. Finally, we require that the mean metallicity increase monotonically with time, with an allowed scatter. We use this age-metallicity prior because SFHs measured from CMDs that do not reach below the oldest main-sequence turnoff suffer from a strong agemetallicity degeneracy (e.g., Weisz et al. 2011). We compute random and systematic uncertainties on the SFH as described in Weisz et al. (2014). Random uncertainties, which are due to the finite number of stars and $\mathrm{S} / \mathrm{N}$ of the $\mathrm{CMD}$, are computed using a Hamiltonian Monte Carlo algorithm as described in Dolphin (2013). Here we ran the chain for $10^{4}$ realizations and use the $68 \%$ confidence interval around the best fit to represent the random uncertainties. Systematic uncertainties, which estimate the sensitivity of the SFH to the choice in underlying stellar models, are computed using 50 Monte Carlo realizations as described in Dolphin (2012) and Weisz et al. (2014).

The derived SFH is shown in Figure 4 and listed in Table 3. Antlia B shows a SFH consistent with the typical dwarf irregular galaxy in the Local Group (e.g., Weisz et al. $2011,2014)$. The results show a relatively constant growth in mass for the first $\sim 10$ Gyr with the last significant rise in star formation occurring $\sim 2-3$ Gyr ago. We discuss the SFH in the context of the other galaxies in the NGC 3109 association in Section 6.

\section{Discussion: Comparison to the NGC 3109 Dwarf Association}

We compare the derived SFH of Antlia B to the other possible members of the NGC 3109 association and to the dwarf galaxy population in the Local Group. All galaxies have had SFHs determined from HST imaging using MATCH, providing a comparison that minimizes systematic uncertainties.

The population of dwarf galaxies in the Local Group can be classified morphologically into two broad classes (see Mateo 1998; Weisz et al. 2011, and references therein): dwarf irregulars (dIs)/dwarf spirals (dSpirals), and dwarf spheroidals (dSphs)/ellipticals (dEs). The first class shows evidence of recent or ongoing star formation and a significant gas reservoir, while the latter shows smooth spatial distributions of stars, no recent star formation, and no significant gas mass.

Studies of nearby dwarf populations have also revealed a small but distinct third morphological class: transition dwarfs (dTrans), which show a high gas fraction like dIs but very little or no recent star formation (Grebel et al. 2003). This lack of recent star formation is often characterized by a lack of $\mathrm{H} \alpha$ emission (Mateo 1998). The origin of this subclass of dwarf galaxies is unclear, but two basic scenarios are proposed. First, dTrans galaxies may be a transitional/intermediate class as dI/ $\mathrm{dSpiral}$ galaxies transform into $\mathrm{dSph} / \mathrm{dE}$ galaxies via physical processes in a group environment (e.g., van Zee et al. 2004a, 2004b). Second, it is possible that we are simply observing the natural duty cycle of star formation in isolated or field dI/dSpiral galaxies (e.g., Skillman et al. 2003; McQuinn et al. 2015a).

The NGC 3109 dwarf association provides an opportunity to explore possible scenarios for dTrans formation and evolution, particularly in an environment well isolated from a massive Milky Way-like host galaxy. A more complete definition of a "dwarf association" and group membership criteria can be found in Tully et al. (2006) and Kourkchi \& Tully (2017). In brief, the NGC 3109 association is the closest group of dwarf galaxies ( $D \sim 1.4 \mathrm{Mpc}$; Tully et al. 2006), which appear to be physically associated but are not expected to be in dynamical equilibrium.

The NGC 3109 association consists of four dI/dSpiral and two dTrans galaxies. The four historic members are NGC 3109 (dSpiral; $\left.M_{V}=-14.9\right)$, Sextans A (dI; $\left.M_{V}=-14.3\right)$, Sextans B (dI; $\left.M_{V}=-14.5\right)$, and the Antlia dwarf (dTrans; $\left.M_{V}=-10.4\right)$. We adopt the morphological classifications from Weisz et al. (2011) and absolute magnitudes from McConnachie (2012). McQuinn et al. (2015b) has suggested that Leo P (dI; $\left.M_{V}=-9.3, D=1.6 \mathrm{Mpc}\right)$ is also likely a member of the association given the similar distance and spatial proximity to the other dwarf galaxies. Antlia B $\left(M_{V}=-9.4\right.$; Table 1) has very similar properties to the Antlia dwarf (e.g., smooth spatial distribution of old stars; gas-rich but no $\mathrm{H} \alpha$ emission indicating a lack of very recent/ongoing star formation), so we classify it as dTrans as well. In total, the association spans a wide absolute magnitude range spread over a large projected area $(\sim 1 \mathrm{Mpc}$ projected spatial extent of the group).

Figures 4(c) and (d) show the HST SFHs for all members of the association: NGC 3109, Antlia (Weisz et al. 2011), Sextans A, Sextans B (Weisz et al. 2014), Leo P (McQuinn et al. 2015b), and Antlia B (this work). We also show the mean 

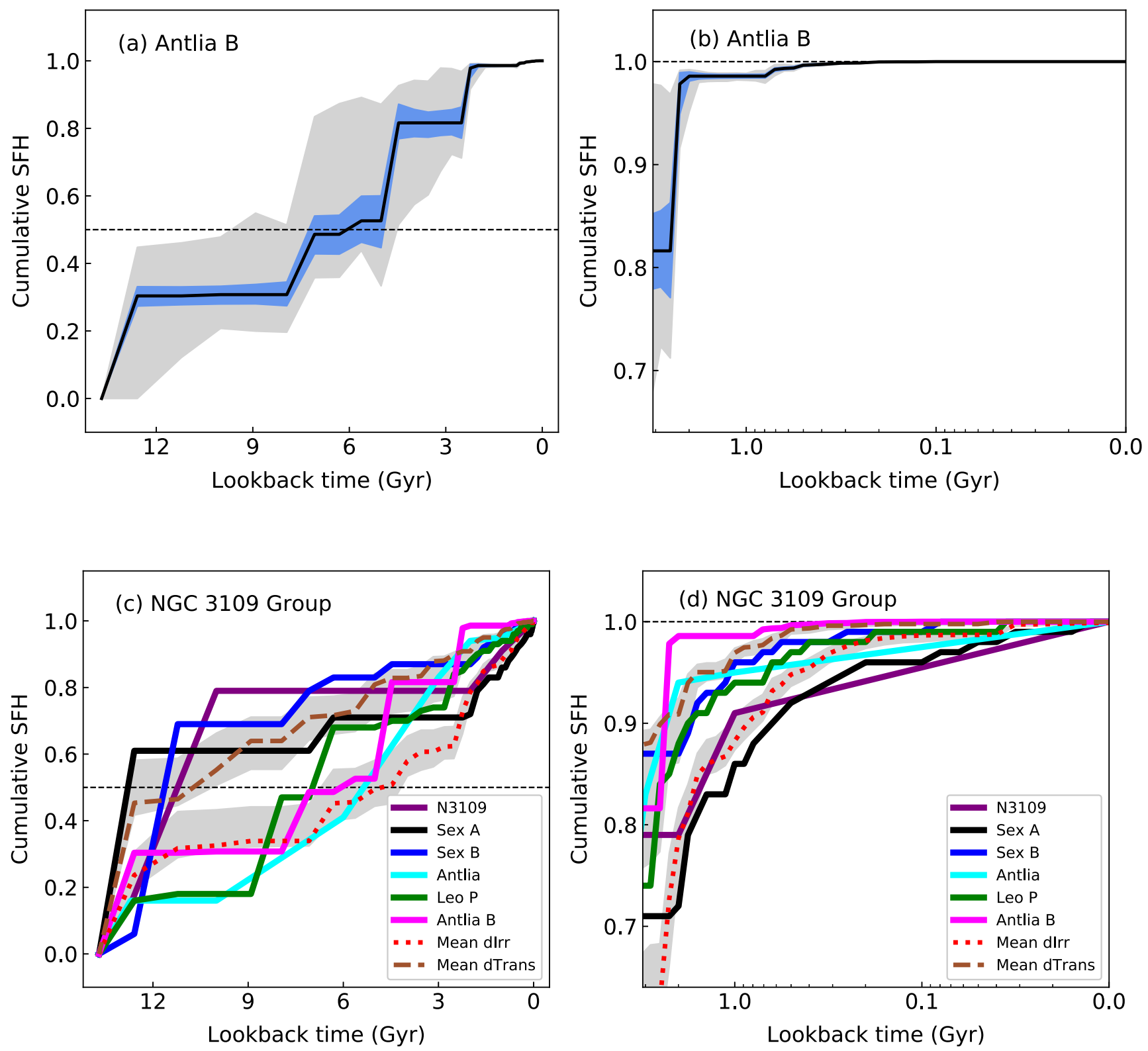

Figure 4. Cumulative SFH for Antlia B (top panels) and the cumulative SFHs for other members of the NGC 3109 dwarf association (bottom panels) taken from the literature. See Section 5 for a description of the analysis and Section 6 for a discussion and relevant references for individual galaxies. The left panels (a), (c) show the complete SFH while the right panels (b), (d) highlight the recent $(t<3 \mathrm{Gyr}) \mathrm{SFH}$. For Antlia B, the blue shading shows the 16\%/84\% confidence regions when accounting for random errors only, while the gray region shows the same confidence intervals when including systematic errors. The sources of random and systematic uncertainty are described in Section 5. For the NGC 3109 group, we show the mean dI and mean dTrans SFHs from Weisz et al. (2014). The gray confidence regions on the mean SFHs reflect the standard error in the mean.

SFH for dI galaxies $(N=8)$ and dTrans $(N=5)$ in the Local Group (from Weisz et al. 2014) as dotted lines. The confidence regions of the mean $\mathrm{dI}$ and dTrans SFHs (shown as gray bands) are the standard error in the mean. Taken together, the NGC 3109 association of dwarfs shows SFHs consistent with the broader dI/dTrans population of the Local Group. If we compare the SFHs of the NGC 3109 association dwarfs to the sample of field dI/dTrans galaxies from Weisz et al. (2014, their Figure 11), we find excellent agreement both in the mean SFH and in the overall spread. There is likely some overlap in samples (i.e., NGC 3109 association galaxies were likely included in the Weisz et al. 2014 sample of isolated galaxies), but the Weisz et al. (2014) sample is clearly larger.

The more luminous members of the NGC 3109 association (NGC 3109, Sextans A, Sextans B) show a slightly more rapid growth in mass than the low-mass systems at early times $(t \gtrsim 9 \mathrm{Gyr}$ ago), perhaps consistent with the expectation that they were born in more massive dark matter halos at early times. Although they appear to track nicely with the mean SFH for dTrans galaxies until $\sim 3 \mathrm{Gyr}$ ago, we note that their general properties are more consistent with dI galaxies.

The three lowest-luminosity systems (Antlia, Antlia B, Leo P) show very similar SFHs, particularly at early times. Consistent with their dTrans classification, the SFHs show that Antlia and Antlia B have formed 95\% or more of their stars in the first $\sim 10 \mathrm{Gyr}$ and track nicely with the mean dTrans SFH within the last $\sim 3 \mathrm{Gyr}$ (Figure 4(d)). Additionally, both Antlia and Antlia B have a $M_{\mathrm{H} \mathrm{I}} / M_{*}$ ratio consistent with other Local Volume dwarf galaxies of similar size (see Figure 3 in Sand et al. 2015), despite the fact that they have no active star 
Table 3

Cumulative Star Formation History of Antlia B

\begin{tabular}{|c|c|c|c|}
\hline $\begin{array}{l}\log (t)(y r) \\
(1)\end{array}$ & $\begin{array}{c}f \\
(2)\end{array}$ & $\begin{array}{c}\sigma_{\operatorname{ran}}(84 \%, 16 \%) \\
(3)\end{array}$ & $\sigma_{\text {tot }}(84 \%, 16 \%)$ \\
\hline 8.60 & 1.00 & $(0.00,0.00)$ & $(0.00,0.00)$ \\
\hline 8.70 & 1.00 & $(0.00,0.00)$ & $(0.00,0.00)$ \\
\hline 8.75 & 0.99 & $(0.00,0.00)$ & $(0.00,0.00)$ \\
\hline 8.80 & 0.99 & $(0.00,0.00)$ & $(0.00,0.00)$ \\
\hline 8.85 & 0.99 & $(0.00,0.00)$ & $(0.00,0.01)$ \\
\hline 8.90 & 0.99 & $(0.00,0.00)$ & $(0.01,0.01)$ \\
\hline 8.95 & 0.99 & $(0.00,0.00)$ & $(0.01,0.00)$ \\
\hline 9.00 & 0.99 & $(0.00,0.00)$ & $(0.00,0.00)$ \\
\hline 9.05 & 0.99 & $(0.00,0.00)$ & $(0.00,0.00)$ \\
\hline 9.10 & 0.99 & $(0.00,0.00)$ & $(0.00,0.01)$ \\
\hline 9.15 & 0.99 & $(0.00,0.00)$ & $(0.00,0.01)$ \\
\hline 9.20 & 0.99 & $(0.00,0.00)$ & $(0.00,0.01)$ \\
\hline 9.25 & 0.99 & $(0.00,0.00)$ & $(0.01,0.01)$ \\
\hline 9.30 & 0.99 & $(0.00,0.00)$ & $(0.01,0.03)$ \\
\hline 9.35 & 0.98 & $(0.01,0.03)$ & $(0.01,0.06)$ \\
\hline 9.40 & 0.82 & $(0.05,0.05)$ & $(0.15,0.10)$ \\
\hline 9.45 & 0.82 & $(0.04,0.04)$ & $(0.16,0.09)$ \\
\hline 9.50 & 0.82 & $(0.04,0.04)$ & $(0.16,0.14)$ \\
\hline 9.55 & 0.82 & $(0.03,0.04)$ & $(0.12,0.21)$ \\
\hline 9.60 & 0.82 & $(0.04,0.04)$ & $(0.13,0.24)$ \\
\hline 9.65 & 0.82 & $(0.06,0.05)$ & $(0.11,0.30)$ \\
\hline 9.70 & 0.53 & $(0.07,0.08)$ & $(0.35,0.19)$ \\
\hline 9.75 & 0.53 & $(0.07,0.06)$ & $(0.37,0.09)$ \\
\hline 9.80 & 0.49 & $(0.06,0.06)$ & $(0.39,0.13)$ \\
\hline 9.85 & 0.49 & $(0.05,0.06)$ & $(0.35,0.13)$ \\
\hline 9.90 & 0.31 & $(0.04,0.03)$ & $(0.21,0.11)$ \\
\hline 9.95 & 0.31 & $(0.03,0.03)$ & $(0.24,0.11)$ \\
\hline 10.00 & 0.31 & $(0.02,0.03)$ & $(0.17,0.10)$ \\
\hline 10.05 & 0.30 & $(0.03,0.03)$ & $(0.16,0.18)$ \\
\hline 10.10 & 0.30 & $(0.03,0.03)$ & $(0.14,0.30)$ \\
\hline 10.14 & 0.00 & $(0.00,0.00)$ & $(0.00,0.00)$ \\
\hline
\end{tabular}

Note. Cumulative star formation history (SFH) for Antlia B (see Section 5). (1) Epoch over which the fractional stellar mass growth $f$ is calculated. (2) Fraction of the total stellar mass formed prior to the corresponding epoch. (3) Upper and lower random uncertainties on the fractional stellar mass. (4) Upper and lower total uncertainties (random and systematic) on the fractional stellar mass.

(This table is available in its entirety in machine-readable form.)

formation like the typical dI galaxy. Leo $\mathrm{P}$, however, shows evidence for active star formation (e.g., O-star embedded in an H II region; Skillman et al. 2013; Evans et al. 2019) consistent with a dI morphological classification.

The spatial proximity of Antlia and Antlia B to NGC 3109 suggests that their nature as dTrans galaxies is due to environmental influences. The Antlia dwarf in particular shows clear evidence of tidal disturbance both in the stellar and gas components. Penny et al. (2012) have shown that Antlia displays stellar tidal tails that are likely the result of an interaction with NGC 3109 (approximately $1 \mathrm{Gyr}$ ago) that may have resulted in the asymmetric H I warp in NGC 3109 (Barnes \& de Blok 2001). In addition, H I gas in Antlia is offset from the main body of the galaxy by $\sim 1^{\prime}$ and aligned with the northwest extension of the stellar tidal tail (Ott et al. 2012).

Although Antlia B shows no evidence of stellar tidal distortion (based on the wide-field imaging analysis by Sand et al. 2015), the relatively close spatial proximity to NGC 3109 suggests that its dTrans properties may also be environmentally driven. The Green Bank Telescope observations of Antlia B described in Sand et al. (2015) were optimized for high- sensitivity detection of $\mathrm{HI}$, but the large beam size $\left(\sim 9^{\prime}\right)$ relative to the small half-light radius of Antlia $\mathrm{B}$ means that no spatial information about the $\mathrm{H} \mathrm{I}$ is available. A future study of Antlia B using high-resolution Very Large Array observations will explore the gas kinematics and distribution in more depth, allowing us to test whether the dTrans properties of this galaxy are environmentally driven.

\section{Conclusions}

In this paper, we present deep HST imaging of the gas-rich, faint dwarf galaxy Antlia B discovered as part of our wide-field imaging survey for satellites of nearby low-mass host galaxies. Our primary results are as follows:

1. We obtain a refined TRGB distance of $D=1.35 \pm$ $0.06 \mathrm{Mpc}\left((m-M)_{0}=25.65 \pm 0.10\right)$ using the HST data (see Section 4). This is slightly more distant than but consistent with the ground-based TRGB determination by Sand et al. (2015). Given the distance and projected separation $(\sim 70 \mathrm{kpc})$, Antlia B is clearly a member of the NGC 3109 dwarf association (Tully et al. 2006; Kourkchi \& Tully 2017).

2. The CMD of Antlia B shows both an old, metal-poor stellar population and a small population of young, more metal-rich stars with ages $\lesssim 1$ Gyr (see Figure 2 ). Consistent with previous ground-based imaging from Sand et al. (2015), we find no evidence for very recent star formation ( 10-100 Myr timescales). The young, blue populations of stars in Antlia B are spatially concentrated toward the galaxy center (see Figure 3).

3. We derive the SFH of Antlia B (see Section 5) using the MATCH algorithm (Dolphin 2002) following the methodology of Weisz et al. (2011, 2014). The SFH is shown in Figure 4 and is consistent with the typical dI/dTrans galaxy in the Local Group. Antlia B shows a slow, constant growth in mass at early times (first 10-11 Gyr). Consistent with a dTrans galaxy classification (see Section 6), Antlia B has had very little star formation since this time despite being relatively gas-rich $\left(M_{\mathrm{H} \mathrm{I}} \sim 3 \times 10^{5} M_{\odot} ;\right.$ Sand et al. 2015). The SFH indicates that only $\sim 1 \%$ of Antlia B's mass formed in the last $\sim 2-3$ Gyr.

4. All members of the NGC 3109 dwarf association have HST-derived SFHs and we present a systematic comparison in Section 6. All six dwarf galaxies-NGC 3109, Sextans A, Sextans B, Antlia, Antlia B, and Leo P-show SFHs consistent with the mean dI/dTrans population in the Local Group (see Figure 4), particularly when considering the isolated field sample of Weisz et al. (2014).

Both Antlia B and Antlia are likely satellites of NGC 3109, as suggested by their spatial proximity, linear distances, and heliocentric systemic HI velocities. Despite the isolated and low-density environment of the NGC 3109 association, the evidence for dynamical interactions between Antlia and NGC 3109 suggests that the suppression of star formation may occur even around very low-mass primary hosts like NGC 3109 (stellar mass $\sim 8 \times 10^{7} M_{\odot}$; McConnachie 2012).

Similar studies to this-which combine uniform, detailed SFH studies based on a spatially complete imaging survey-are currently rare in dwarf association/group environments, but are essential for building a more complete picture of dwarf galaxy evolution. For example, given the small numbers of galaxies in 
the NGC 3109 group, finding weak trends in SFHs with properties like luminosity, morphology, and/or radial distance from a primary host are inevitable. More studies of dwarf associations will not only provide insights into possible correlations but will allow for broader studies into the role of environment in shaping the SFHs of the lowest-luminosity dwarf satellites.

We would like to thank the referee for the excellent feedback that significantly improved this paper. J.R.H. acknowledges support from HST award GO-14078 and the hospitality of the Texas Tech University Department of Physics and Astronomy and the University of Arizona Department of Astronomy/ Steward Observatory. S.M.A. is supported by the National Science Foundation Graduate Research Fellowship under Grant DGE 1752814. Research by D.J.S. is supported by NSF grants AST-1821967, 1821987, 1813708, and 1813466. Research by D.C. is supported by NSF grant AST-1814208, and by NASA through grants No. HST-GO-15426.007-A and HST-GO-HSTGO-15332.004-A from the Space Telescope Science Institute, which is operated by AURA, Inc., under NASA contract NAS 5-26555. K.S. acknowledges support from the Natural Sciences and Engineering Research Council of Canada. B.W. and J.C. were supported by an NSF Faculty Early Career Development (CAREER) award (AST-1151462). D.R.W. acknowledges fellowships from the Alfred P. Sloan Foundation and the Alexander von Humboldt Foundation. A.H.G.P. is supported by NSF grant AST-1813628. This work was partially performed at the Aspen Center for Physics, which is supported by National Science Foundation grant PHY-1607611.

Facility: HST.

Software: astropy (Astropy Collaboration et al. 2013, 2018), MATCH (Dolphin 2002).

\section{ORCID iDs}

J. R. Hargis (iD https://orcid.org/0000-0002-8722-9806

S. Albers (iD https://orcid.org/0000-0001-5496-2668

D. Crnojević (iD https://orcid.org/0000-0002-1763-4128

D. J. Sand (iD https://orcid.org/0000-0003-4102-380X

D. R. Weisz (i) https://orcid.org/0000-0002-6442-6030

J. L. Carlin (iD https://orcid.org/0000-0002-3936-9628

K. Spekkens (i) https://orcid.org/0000-0002-0956-7949

B. Willman (i) https://orcid.org/0000-0003-2892-9906

A. H. G. Peter (i) https://orcid.org/0000-0002-8040-6785

A. E. Dolphin (iD https://orcid.org/0000-0001-8416-4093

\section{References}

Astropy Collaboration, Price-Whelan, A. M., Sipőcz, B. M., et al. 2018, AJ, 156,123

Astropy Collaboration, Robitaille, T. P., Tollerud, E., et al. 2013, A\&A, 558, A33

Barnes, D. G., \& de Blok, W. J. G. 2001, AJ, 122, 825

Bellazzini, M., Beccari, G., Fraternali, F., et al. 2014, A\&A, 566, A44

Bennet, P., Sand, D. J., Crnojević, D., et al. 2017, ApJ, 850, 109

Bennet, P., Sand, D. J., Crnojević, D., et al. 2019, ApJ, 885, 153

Brooks, A. M., Kuhlen, M., Zolotov, A., \& Hooper, D. 2013, ApJ, 765, 22

Bullock, J. S., \& Boylan-Kolchin, M. 2017, ARA\&A, 55, 343

Carlin, J. L., Garling, C. T., Peter, A. H. G., et al. 2019, ApJ, 886, 109

Carlin, J. L., Sand, D. J., Price, P., et al. 2016, ApJL, 828, L5
Carrillo, A., Bell, E. F., Bailin, J., et al. 2017, MNRAS, 465, 5026

Chiboucas, K., Karachentsev, I. D., \& Tully, R. B. 2009, AJ, 137, 3009

Choi, J., Dotter, A., Conroy, C., et al. 2016, ApJ, 823, 102

Crnojević, D., Sand, D. J., Bennet, P., et al. 2019, ApJ, 872, 80

Crnojević, D., Sand, D. J., Caldwell, N., et al. 2014, ApJL, 795, L35

Crnojević, D., Sand, D. J., Spekkens, K., et al. 2016, ApJ, 823, 19

Dalcanton, J. J., Williams, B. F., Seth, A. C., et al. 2009, ApJS, 183, 67

Danieli, S., van Dokkum, P., Merritt, A., et al. 2017, ApJ, 837, 136

Dohm-Palmer, R. C., Skillman, E. D., Mateo, M., et al. 2002, AJ, 123, 813

Dolphin, A. E. 2000, PASP, 112, 1383

Dolphin, A. E. 2002, MNRAS, 332, 91

Dolphin, A. E. 2012, ApJ, 751, 60

Dolphin, A. E. 2013, ApJ, 775, 76

Dolphin, A. E., Saha, A., Skillman, E. D., et al. 2003, AJ, 126, 187

Dooley, G. A., Peter, A. H. G., Carlin, J. L., et al. 2017, MNRAS, 472, 1060

Dotter, A., Chaboyer, B., Jevremović, D., et al. 2008, ApJS, 178, 89

Evans, C. J., Castro, N., Gonzalez, O. A., et al. 2019, A\&A, 622, A129

Ford, H. C., Bartko, F., Bely, P. Y., et al. 1998, Proc. SPIE, 3356, 234

Gatto, A., Fraternali, F., Read, J. I., et al. 2013, MNRAS, 433, 2749

Geha, M., Wechsler, R. H., Mao, Y.-Y., et al. 2017, ApJ, 847, 4

Girardi, L., Williams, B. F., Gilbert, K. M., et al. 2010, ApJ, 724, 1030

Grebel, E. K., Gallagher, J. S., III, \& Harbeck, D. 2003, AJ, 125, 1926

Hidalgo, S. L., Aparicio, A., \& Gallart, C. 2008, AJ, 136, 2332

Jang, I. S., \& Lee, M. G. 2017, ApJ, 835, 28

Kallivayalil, N., Sales, L., Zivick, P., et al. 2018, ApJ, 867, 19

Kim, S. Y., Peter, A. H. G., \& Hargis, J. R. 2018, PhRvL, 121, 211302

Koposov, S. E., Walker, M. G., Belokurov, V., et al. 2018,

Kourkchi, E., \& Tully, R. B. 2017, ApJ, 843, 16

Kroupa, P. 2001, MNRAS, 322, 231

Lee, M. G., Freedman, W. L., \& Madore, B. F. 1993, ApJ, 417, 553

Madore, B. F., Mager, V., \& Freedman, W. L. 2009, ApJ, 690, 389

Makarov, D., Makarova, L., Rizzi, L., et al. 2006, AJ, 132, 2729

Marigo, P., Girardi, L., Bressan, A., et al. 2017, ApJ, 835, 77

Mateo, M. L. 1998, ARA\&A, 36, 435

McConnachie, A. W. 2012, AJ, 144, 4

McQuinn, K. B. W., Cannon, J. M., Dolphin, A. E., et al. 2015a, ApJ, 802, 66 McQuinn, K. B. W., Skillman, E. D., Cannon, J. M., et al. 2010, ApJ, 721, 297 McQuinn, K. B. W., Skillman, E. D., Dolphin, A., et al. 2015b, ApJ, 812, 158 McQuinn, K. B. W., Skillman, E. D., Dolphin, A. E., Berg, D., \& Kennicutt, R. 2016, ApJ, 826, 21

Minniti, D., Zijlstra, A. A., \& Alonso, M. V. 1999, AJ, 117, 881

Ott, J., Stilp, A. M., Warren, S. R., et al. 2012, AJ, 144, 123

Penny, S. J., Pimbblet, K. A., Conselice, C. J., et al. 2012, ApJL, 758, L32

Rizzi, L., Tully, R. B., Makarov, D., et al. 2007, ApJ, 661, 815

Sakai, S., Madore, B. F., Freedman, W. L., et al. 1997, ApJ, 478, 49

Sales, L. V., Navarro, J. F., Kallivayalil, N., \& Frenk, C. S. 2017, MNRAS, 465,1879

Sand, D. J., Crnojević, D., Strader, J., et al. 2014, ApJL, 793, L7

Sand, D. J., Spekkens, K., Crnojević, D., et al. 2015, ApJL, 812, L13

Sawala, T., Frenk, C. S., Fattahi, A., et al. 2016, MNRAS, 457, 1931

Schlafly, E. F., \& Finkbeiner, D. P. 2011, ApJ, 737, 103

Schlegel, D. J., Finkbeiner, D. P., \& Davis, M. 1998, ApJ, 500, 525

Skillman, E. D., Monelli, M., Weisz, D. R., et al. 2017, ApJ, 837, 102

Skillman, E. D., Salzer, J. J., Berg, D. A., et al. 2013, AJ, 146, 3

Skillman, E. D., Tolstoy, E., Cole, A. A., et al. 2003, ApJ, 596, 253

Smercina, A., Bell, E. F., Price, P. A., et al. 2018,

Smercina, A., Bell, E. F., Slater, C. T., et al. 2017, ApJL, 843, L6

Stinson, G. S., Dalcanton, J. J., Quinn, T., et al. 2009, MNRAS, 395, 1455

Toloba, E., Sand, D. J., Spekkens, K., et al. 2016, ApJL, 816, L5

Tolstoy, E., Hill, V., \& Tosi, M. 2009, ARA\&A, 47, 371

Torrealba, G., Belokurov, V., Koposov, S. E., et al. 2018, MNRAS, 475, 5085

Tully, R. B., Rizzi, L., Dolphin, A. E., et al. 2006, AJ, 132, 729

van Dyk, S. D., Puche, D., \& Wong, T. 1998, AJ, 116, 2341

van Zee, L., Barton, E. J., \& Skillman, E. D. 2004a, AJ, 128, 2797

van Zee, L., Skillman, E. D., \& Haynes, M. P. 2004b, AJ, 128, 121

VandenBerg, D. A., Bergbusch, P. A., Ferguson, J. W., \& Edvardsson, B. 2014, ApJ, 794, 72

Weisz, D. R., Dalcanton, J. J., Williams, B. F., et al. 2011, ApJ, 739, 5

Weisz, D. R., Dolphin, A. E., Skillman, E. D., et al. 2014, ApJ, 789, 147

Wetzel, A. R., Hopkins, P. F., Kim, J.-h., et al. 2016, ApJL, 827, L23

Williams, B. F., Lang, D., Dalcanton, J. J., et al. 2014, ApJS, 215, 9 